ఠ

\title{
Anti-inflammatory effects of a topical preparation containing nicotinamide, retinol, and 7-dehydrocholesterol in patients with acne: a gene expression study
}

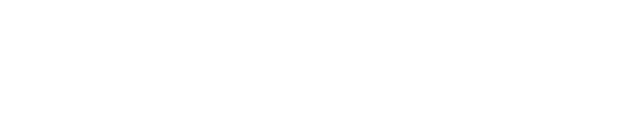

Enzo Emanuele'

Marco Bertona'

Karmela Altabas ${ }^{2}$

Velimir Altabas ${ }^{2}$

Giuseppe Alessandrini ${ }^{3}$

'Department of Health Sciences, University of Pavia, Pavia,

Italy; ${ }^{2}$ Clinical Hospital "Sestre

Milosrdnice”, Zagreb, Croatia;

${ }^{3}$ Dermatology Clinics, Ugento, Italy
Correspondence: Enzo Emanuele Department of Health Sciences, University of Pavia, Via Bassi,

2I, I-27I00, Pavia, Italy

Tel +393385054463

Fax +390384453II97

Email enzo.em@libero.it
Purpose: Acne vulgaris is a skin disorder of the sebaceous follicles, involving hyperkeratinization and perifollicular inflammation. Aberrant extracellular matrix remodeling due to matrix metalloproteinases (MMPs) has been associated with the presence of acne conditions. Given the complex pathophysiology of acne, novel topical therapies should include combination products that target multiple pathogenetic mechanisms. In this pilot study we investigated the changes in gene expression of extracellular MMPs, the tissue inhibitors of metalloproteinases, and proinflammatory molecules after 45 days of topical application of a combination product containing nicotinamide, retinol, and 7-dehydrocholesterol in 16 patients with inflammatory acne on their back.

Materials and methods: Skin biopsies were obtained before and after treatment for gene expression studies.

Results: Quantitative real-time polymerase chain reaction revealed a significant downregulation of $M M P-1, M M P-2, M M P-9, M M P-14$, interleukin-6, monocyte chemoattractant protein-1, and macrophage migration inhibitory factor. In contrast, the tissue inhibitors of metalloproteinases and transforming growth factor- $\beta 1$ were significantly upregulated. The gene expression findings correlated well with the clinical treatment response.

Conclusions: The combination of nicotinamide, retinol, and 7-dehydrocholesterol appears to be effective for acne treatment from both clinical and molecular standpoints.

Keywords: acne, gene expression, topical treatment, matrix metalloproteinases, inflammation

\section{Introduction}

Acne vulgaris is a common chronic inflammatory cutaneous disease involving the pilosebaceous unit. ${ }^{1-3}$ Epidemiological estimates suggest that acne can affect up to 50.9 percent of women and 42.5 percent of men throughout their twenties and may continue to occur throughout adulthood. ${ }^{4}$ Acne is a complex multifactorial condition with several pathogenic factors. In general, acne is considered to involve excess sebum production and hyperplasia of the sebaceous glands, the subsequent formation of microcomedones associated with the hyperkeratinization of the follicular wall, and the induction of inflammatory reactions in keratinocytes and sebocytes with invaded inflammatory cells. ${ }^{5-7}$ Of note, Propionibacterium acnes (P. acnes) - a Gram-positive anaerobic microbial species found in sebum-rich skin - is considered to perpetuate the pathogenetic process of acne through the induction of proinflammatory and chemotactic molecules. ${ }^{8,9}$ 
In recent years, molecular studies have shown a key role for aberrant extracellular matrix remodeling in the pathogenesis of acne. This process is regulated by matrix metalloproteinases (MMPs) and their inhibitor enzymes, the tissue inhibitors of metalloproteinases (TIMPs). ${ }^{10}$ In a landmark study, Kang et $\mathrm{al}^{11}$ demonstrated that transcription factors nuclear factor-kappa B and activator protein-1 are activated in acne lesions, ultimately leading to an increased expression of inflammatory cytokines and MMPs. In line with these findings, Papakonstantinou et $\mathrm{al}^{12}$ have shown that MMPs and TIMPs of epithelial origin are involved in acne pathogenesis, and that treatment with isotretinoin reduces the expression of $M M P-9$ and $M M P-13$ in keratinocytes. Using gene array expression profiling, Trivedi et $\mathrm{a}^{13}$ reported that most of the genes dysregulated in acne are involved in the inflammation and extracellular matrix remodeling pathways. Choi et $\mathrm{al}^{14}$ have shown that the expression of proMMP-2 is induced by $P$. acnes through the nuclear factor-kappa B pathway.

Most of the conventional therapeutic agents currently used in the management of acne are designed to hit a single pathophysiological target. ${ }^{15}$ Unfortunately, the physiological and mechanistic deregulations responsible for acne initiation and perpetuation implicate a number of genes or signaling cascades so that it appears evident that multitargeted approaches are requested to overcome this skin disorder. Growing evidence highlights the importance of topical combination products targeting multiple pathogenic factors. ${ }^{16}$ Due to their complementary and synergistic mechanisms of action, combination products hold great promise for a comprehensive and multitargeted treatment approach in acne. Because nicotinamide, ${ }^{17}$ retinol, ${ }^{18}$ and 7 -dehydrocholesterol ${ }^{19}$ have shown anti-inflammatory and immunomodulatory effects at the skin level, in this study we sought to analyze the changes in gene expression of extracellular MMPs, TIMPs, and proinflammatory molecules after 45 days of application of a combination product containing these actives in a topical form. The study of gene expression changes in response to a topical combination treatment may be useful to gain insights into how inflammatory responses and matrix remodeling may reflect the observed modifications in skin appearance.

\section{Materials and methods}

\section{Study participants}

The study population comprised 16 Caucasian patients aged $>18$ years (five men and eleven women; mean age: 27.7 years \pm 5.1 years) with active inflammatory acne on their back. Subjects were excluded if they had previously received oral antibiotics, benzoyl peroxide, tretinoin, and oral retinoids. In addition, patients with endocrine disease, diabetes mellitus, or severe physical illnesses or those who were currently using oral contraceptives, implantable contraceptives, prednisone, or other steroids were not eligible for participation. This study has been approved by the local ethics committee and conforms to the Declaration of Helsinki. Before the study, each participant was informed about the purpose of the study, and signed informed consents were obtained.

\section{Materials}

Test materials were supplied by Biodue S.p.A. (Tavarnelle Val Di Pesa, Italy). The topical preparation tested in this study contained nicotinamide (4\% weight/weight), retinol ( $1 \%$ weight/weight), and 7-dehydrocholesterol ( $0.5 \%$ weight/ weight) in a moisturizer base. The concentrations of the actives were fixed based on previous studies. ${ }^{20-22}$

\section{Procedures}

All participants were asked to withdraw any topical product 14 days before the beginning of the study. In addition, they were not allowed to use any topical intervention throughout the entire study period. The subjects applied the combination product twice per day (morning and evening) for a total of 45 days. After the baseline visit (Day 0), patients were instructed to apply the combination product over the acne areas on the back twice per day (once in the morning and once in the evening) and were then assessed at follow-up visit on Day 45. In line with previous methodology, ${ }^{23}$ the clinical criterion for efficacy was the investigator's global improvement rating on a five-point scale ( -1 , worsened; 0 , unchanged; 1 , improved; 2, markedly improved; and 3 , resolved). Tolerance was assessed by asking patients about any signs or symptoms of adverse reactions.

\section{Skin biopsies and gene selection}

Paired skin specimens from acne areas were obtained on Day 0 and Day 45 through $4 \mathrm{~mm}$ punch biopsies for ribonucleic acid (RNA) extraction and molecular analyses. Based on the current knowledge of the pathophysiology of acne, ${ }^{1-6}$ the following genes were selected for the expression study: $M M P-1 ; M M P-2 ; M M P-3 ; M M P-7 ; M M P-$ 8; MMP-9; MMP-12; MMP-13; MMP-14; TIMP-1; TIMP-2; TIMP-3; transforming growth factor- $\beta$ (TGF- $\beta$ ); interleukin-6 (IL-6); monocyte chemoattractant protein-1 (MCP-1); macrophage migration inhibitory factor (MIF); and regulated upon activation, normally T-cell expressed, and presumably secreted (RANTES). 


\section{Quantitative real-time polymerase chain reaction}

RNA from baseline and post-treatment skin samples was isolated using the RNeasy Mini Kit (Qiagen, Valencia, $\mathrm{CA}$ ) according to the manufacturer's instructions. Integrity of RNA was assessed with agarose gel electrophoresis, and RNA quantity was measured by spectrophotometry. A $1 \mu \mathrm{g}$ amount of RNA was reverse transcribed using the iScript cDNA Synthesis Kit (BioRad, Hercules, CA) according to the manufacturer's instructions. cDNA was stored at $-20^{\circ} \mathrm{C}$. All quantitative real-time polymerase chain reactions (qRT-PCR) were carried out on a BioRad iQ5 Cycler (BioRad). In brief, a $25 \mu \mathrm{L}$ reaction solution consisted of iQ SYBR Green Supermix (BioRad), forward and reverse primers (final concentration $400 \mathrm{nM}$ each), and cDNA mixture (40 ng). The conditions for qRT-PCR were as follows: preheating at $95^{\circ} \mathrm{C}$ for 10 minutes, followed by 40 cycles of $95^{\circ} \mathrm{C}$ for 10 seconds, $60^{\circ} \mathrm{C}$ for 15 seconds, and $72^{\circ} \mathrm{C}$ for 30 seconds. Melting curves were analyzed for each reaction by steadily increasing the temperature from $60^{\circ} \mathrm{C}$ to $95^{\circ} \mathrm{C}$. Fluorescence data were analyzed using the BioRad iQ5 Optical System Software Version 2.0. To control for variations in RNA quality and quantity, the expression of the gene of interest was normalized to the expression of hypoxanthine phosphoribosyltransferase-1 (HPRT1) as a housekeeping gene. mRNA expression levels were calculated according to the following formula: $2^{-\triangle C T}$, where $\Delta \mathrm{CT}$ (sample) was defined as CT (gene of interest) - CT (HPRT1). Nucleotide sequences of primers used for qRTPCR reactions are available upon request.

\section{Statistical analysis}

All calculations were performed using SPSS 17.0 software (SPSS, Inc, Chicago, IL). Pre- and post-treatment gene expression data were analyzed using paired $t$-tests after adjustment for age and sex. A two-tailed $P$-value $<0.05$ was considered statistically significant.

\section{Results}

In this study a total of 16 paired skin samples from acne areas on the back were obtained before and after 45 days of application of a topical combination product and then subjected to gene expression studies. Assessment of efficacy by the investigators showed that $94 \%$ of the patients had improved after 45 days of treatments, while $6 \%$ demonstrated an unchanged state (Table 1). No patient discontinued treatment due to adverse effects.
Table I Physician's overall ratings for the response of inflammatory acne after 45 days of topical application of a combination product containing nicotinamide, retinol, and 7-dehydrocholesterol in 16 patients with inflammatory acne on their back

\begin{tabular}{ll}
\hline Rating & Number (\%) \\
\hline Worsened & $0(0 \%)$ \\
Unchanged & $\mathrm{I}(6 \%)$ \\
Improved & $10(63 \%)$ \\
Markedly improved & $4(25 \%)$ \\
Resolved & $\mathrm{I}(6 \%)$ \\
Total & $16(100 \%)$ \\
\hline
\end{tabular}

In order to reveal potential treatment-related changes in gene expression in areas with acne lesions, qRT-PCRbased expression analyses of 17 genes were performed. All examined genes were selected due to their potential relevance in inflammation and matrix remodeling under acne conditions. Expression analysis was successful in all skin biopsies. qRT-PCR data concerning MMPs and TIMPs (Table 2) revealed a significant downregulation of $M M P-1$, $M M P-2, M M P-9$, and $M M P-14$ after 45 days of treatment compared with baseline values. In addition, TIMPs and TGF- $\beta$ were significantly upregulated by the topical combination treatment. The expression of other MMPS and TIMPs genes was unchanged (data not shown).

Table 3 shows the changes in inflammatory gene expression. The results indicated that $I L-6, M C P-1$, and $M I F$ were significantly downregulated by topical treatment, whereas no significant changes in the expression of RANTES were detected.

Table 2 Relative gene expression values (MMPs and their inhibitors) before and after 45 days of topical application of a combination product containing nicotinamide, retinol, and 7-dehydrocholesterol in 16 patients with inflammatory acne on their back

\begin{tabular}{lccl}
\hline & Baseline & 45 days & $\boldsymbol{P}$ \\
\hline MMP-I & $338 \pm 77$ & $211 \pm 41$ & $<0.001$ \\
MMP-2 & $728 \pm 184$ & $461 \pm 191$ & $<0.001$ \\
MMP-9 & $1.4 \pm 0.3$ & $0.6 \pm 0.2$ & $<0.001$ \\
MMP-I & $378 \pm 91$ & $256 \pm 53$ & $<0.001$ \\
TGF- $\beta$ & $88 \pm 11$ & $146 \pm 26$ & $<0.001$ \\
TIMP-I & $2918 \pm 846$ & $6483 \pm 1091$ & $<0.001$ \\
TIMP-2 & $212 \pm 67$ & $501 \pm 117$ & $<0.001$ \\
TIMP-3 & $381 \pm 118$ & $663 \pm 193$ & $<0.001$ \\
\hline
\end{tabular}

Notes: Messenger ribonucleic acid expression levels were calculated according to the formula 2- ${ }^{-\Delta C}$, where $\Delta C T$ (sample) was defined as CT (gene of interest) - CT (HPRTI). Only significant values after adjustment for age and sex are shown. Abbreviations: MMP, matrix metalloproteinase; TGF, transforming growth factor; TIMP, tissue inhibitor of metalloproteinases. 
Table 3 Relative gene expression values (inflammatory genes) before and after 45 days of topical application of a combination productcontaining nicotinamide, retinol, and7-dehydro-cholesterol in 16 patients with inflammatory acne on their back

\begin{tabular}{lcll}
\hline & Baseline & 45 days & $\boldsymbol{P}$ \\
\hline IL-6 & $1775 \pm 867$ & $944 \pm 569$ & $<0.00$ I \\
MCP-I & $544 \pm 18$ I & $225 \pm 86$ & $<0.00$ I \\
MIF & $358 \pm 117$ & $265 \pm 98$ & $<0.01$ \\
\hline
\end{tabular}

Notes: Messenger ribonucleic acid expression levels were calculated according to the formula $2^{-\Delta C T}$, where $\Delta C T$ (sample) was defined as CT (gene of interest) - CT (HPRTI). Only significant values after adjustment for age and sex are shown.

Abbreviations: MCP, monocyte chemoattractant protein; MIF, migration inhibitory factor; IL-6, interleukin-6.

\section{Discussion}

The results of this study support the safety and efficacy of a combination product containing nicotinamide, retinol, and 7-dehydrocholesterol in the treatment of acne. More importantly, we have shown by molecular analysis that the topical application of this combination resulted in a significant downregulation of $M M P-1, M M P-2, M M P-9, M M P-14, I L-6$, $M C P-1$, and $M I F$ in acne areas. In contrast, both TIMPs and $T G F-\beta$ were significantly upregulated.

Topical application of nicotinamide has a stabilizing effect on epidermal barrier function, as reflected by a reduction in transepidermal water loss and by an improvement in the moisture content of the horny layer. ${ }^{24}$ Retinol (preformed vitamin A) plays a crucial role in cell growth and differentiation of human epithelial tissues. ${ }^{25}$ The combined use of nicotinamide, retinol, and 7-dehydrocholesterol in acne has not been previously studied either clinically or at the molecular level. 7-dehydrocholesterol is the main precursor of vitamin D - a key modulator of keratinocyte differentiation and proliferation - in the skin. ${ }^{19}$ In addition, specific vitamin D derivatives may exhibit comedolytic activity. ${ }^{26}$ The results of our molecular analyses indicate that the combination of nicotinamide, retinol, and 7-dehydrocholesterol significantly decreased $M M P$ expression and increased TIMP expression. In addition, we have also demonstrated a significant upregulation of $T G F-\beta$, a master regulator of the expression of MMPs and extracellular matrix remodeling. ${ }^{27}$ An imbalance in the ratio of MMPs to TIMPs has been suggested to play a key role in the development of atrophic or hypertrophic scars in patients with acne. ${ }^{28}$ As prevention is the main step in avoiding the appearance of postacne scars, ${ }^{28}$ our gene expression data seem to suggest that a combined treatment approach may have value in preventing the disfiguring consequences of acne.

Inflammation is involved in acne conditions by recruiting leukocytes and promoting extracellular matrix remodeling. ${ }^{3}$ We found that the expression of three inflammatory genes
(IL-6, MCP-1, and MIF) was significantly downregulated in areas with acne by topical treatment. IL-6 is a cytokine with multiple and complex proinflammatory effects and is produced primarily by macrophages, T cells, endothelial cells, and fibroblasts. ${ }^{29}$ The major biological effects of IL-6 also include stimulation of the proliferation and differentiation of $\mathrm{T}$ lymphocytes and regulation of the acute-phase response. ${ }^{29}$ IL-6 can play a role as a regulator of extracellular matrix deposition and is involved in the immune response to $P$. acnes; as a consequence, it may be an important determinant of acne. ${ }^{30} \mathrm{MCP}-1$ is a chemokine responsible for the recruitment of monocytes to sites of inflammation and is related to the extent of macrophage infiltration into the skin. ${ }^{31}$ MIF is an integral component of the host antimicrobial alarm system and stress response that promotes the proinflammatory functions of immune cells. ${ }^{32}$ Taken together, these results demonstrate that the combination of nicotinamide, retinol, and 7-dehydrocholesterol may be useful not only to prevent matrix remodeling but also to inhibit the inflammatory reactions that are paramount in the clinical manifestations of acne.

There are several limitations of this study that need to be mentioned. First, we performed gene expression studies in acne areas of the back. Therefore, caution is needed in the extrapolation of our findings to other skin areas. Second, this study was conducted in Caucasian individuals, so results cannot be simply extrapolated to populations with different ethnic backgrounds. Third, our study should be considered an exploratory analysis, and further data on protein levels are needed to extend and confirm our results. Although we showed that a combination treatment is effective in modulating the main molecular alterations associated with acne, our study did not compare the effect of the combination product with that of each compound alone (i.e., nicotinamide, retinol, and 7-dehydrocholesterol alone). Our study was not designed as a comparative trial of different treatment strategies. Acne patients should most likely be treated on an individual basis according to each patient's disease characteristics, based on clinical trial data and influenced by the personal experience of the physician. In the future, prospective evaluation of combination versus simple or sequential single-agent therapy may help identify optimal treatment approaches. Another limitation that should be kept in mind is that the regulation of gene expression does not invariably correlate with protein expression levels, because molecular mechanisms of transcriptional pathways differ from those of translation ones. Future studies using immunohistochemical staining and/or Western blotting are needed to corroborate our findings. Finally, the lack of a control arm makes our data exploratory in nature. 


\section{Conclusions}

Notwithstanding the pilot nature of the study, our data support at the molecular level the safety and efficacy of a combination product containing nicotinamide, retinol, and 7-dehydrocholesterol in the treatment of acne. This approach seems to be effective in restoring the unbalanced extracellular matrix remodeling and the inflammatory component, which are paramount to the pathogenesis of acne.

\section{Disclosure}

The authors report no conflicts of interest in this work.

\section{References}

1. Ingram JR, Grindlay DJ, Williams HC. Management of acne vulgaris: an evidence-based update. Clin Exp Dermatol. 2010;35:351-354.

2. Wang KC, Zane LT. Recent advances in acne vulgaris research: insights and clinical implications. Adv Dermatol. 2008;24:197-209.

3. Taylor M, Gonzalez M, Porter R. Pathways to inflammation: acne pathophysiology. Eur J Dermatol. 2011;21:323-333.

4. Collier C, Harper JC, Cafardi JA, et al. The prevalence of acne in adults 20 years and older. J Am Acad Dermatol. 2008;58:56-59.

5. Williams HC, Dellavalle RP, Garner S. Acne vulgaris. Lancet. 2012;379:361-372.

6. Harper JC, Thiboutot DM. Pathogenesis of acne: recent research advances. Adv Dermatol. 2003;19:1-10.

7. Ayer J, Burrows N. Acne: more than skin deep. Postgrad Med J. 2006;82:500-506.

8. Dessinioti C, Katsambas AD. The role of Propionibacterium acnes in acne pathogenesis: facts and controversies. Clin Dermatol. 2010;28:2-7.

9. Perry AL, Lambert PA. Propionibacterium acnes. Lett Appl Microbiol. 2006;42:185-188.

10. Philips N, Auler S, Hugo R, et al. Beneficial regulation of matrix metalloproteinases for skin health. Enzyme Res. 2011;2011:427285.

11. Kang S, Cho S, Chung JH, et al. Inflammation and extracellular matrix degradation mediated by activated transcription factors nuclear factorkappaB and activator protein-1 in inflammatory acne lesions in vivo. Am J Pathol. 2005;166:1691-1699.

12. Papakonstantinou E, Aletras AJ, Glass E, et al. Matrix metalloproteinases of epithelial origin in facial sebum of patients with acne and their regulation by isotretinoin. J Invest Dermatol. 2005;125:673-684.

13. Trivedi NR, Gilliland KL, Zhao W, et al. Gene array expression profiling in acne lesions reveals marked upregulation of genes involved in inflammation and matrix remodeling. J Invest Dermatol. 2006;126: 1071-1079.

14. Choi JY, Piao MS, Lee JB, et al. Propionibacterium acnes stimulates pro-matrix metalloproteinase-2 expression through tumor necrosis factor-alpha in human dermal fibroblasts. $J$ Invest Dermatol. 2008;128:846-854.
15. Kraft J, Freiman A. Management of acne. CMAJ. 2011;183:E430-E435.

16. Tanghetti EA. Combination therapy is the standard of care. Cutis. 2005;76:8-14.

17. Grange PA, Raingeaud J, Calvez V, Dupin N. Nicotinamide inhibits Propionibacterium acnes-induced IL-8 production in keratinocytes through the NF-kappaB and MAPK pathways. J Dermatol Sci. 2009;56:106-112.

18. Ruamrak C, Lourith N, Natakankitkul S. Comparison of clinical efficacies of sodium ascorbyl phosphate, retinol and their combination in acne treatment. Int J Cosmet Sci. 2009;31:41-46.

19. Glossmann HH. Origin of 7-dehydrocholesterol (provitamin D) in the skin. J Invest Dermatol. 2010;130:2139-2141.

20. Iraji F, Banan L. The efficacy of nicotinamide gel $4 \%$ as an adjuvant therapy in the treatment of cutaneous erosions of pemphigus vulgaris. Dermatol Ther. 2010;23:308-311.

21. Ho ET, Trookman NS, Sperber BR, et al. A randomized, doubleblind, controlled comparative trial of the anti-aging properties of nonprescription tri-retinol $1.1 \%$ vs prescription tretinoin $0.025 \%$. J Drugs Dermatol. 2012;11:64-69.

22. Seifert M, Tilgen W, Reichrath J. Expression of 25-hydroxyvitamin D-1alpha-hydroxylase (1alphaOHase, CYP27B1) splice variants in $\mathrm{HaCaT}$ keratinocytes and other skin cells: modulation by culture conditions and UV-B treatment in vitro. Anticancer Res. 2009;29:3659-3667.

23. Konishi N, Endo H, Oiso N, Kawara S, Kawada A. Acne phototherapy with a 1450-nm diode laser: an open study. Ther Clin Risk Manag. 2007;3:205-209.

24. Namazi MR. Nicotinamide in dermatology: a capsule summary. Int $J$ Dermatol. 2007;46:1229-1231.

25. Amann PM, Schadendorf D, Owen RW, et al. Retinal and retinol are potential regulators of gene expression in the keratinocyte cell line HaCaT. Exp Dermatol. 2011;20:373-375.

26. Nieves NJ, Ahrens JM, Plum LA, et al. Identification of a unique subset of 2-methylene-19-nor analogs of vitamin D with comedolytic activity in the rhino mouse. J Invest Dermatol. 2010;130:2359-2367.

27. Kim J. Review of the innate immune response in acne vulgaris: activation of Toll-like receptor 2 in acne triggers inflammatory cytokine responses. Dermatology. 2005;211:193-198.

28. Fabbrocini G, Annunziata MC, D'Arco V, et al. Acne scars: pathogenesis, classification and treatment. Dermatol Res Pract. 2010;2010:893080.

29. Scheller J, Chalaris A, Schmidt-Arras D, et al. The pro- and antiinflammatory properties of the cytokine interleukin-6. Biochim Biophys Acta. 2011;1813:878-888.

30. Pajulo OT, Pulkki KJ, Alanen MS, et al. Correlation between interleukin-6 and matrix metalloproteinase-9 in early wound healing in children. Wound Repair Regen. 1999;7:453-457.

31. Deshmane SL, Kremlev S, Amini S, et al. Monocyte chemoattractant protein-1 (MCP-1): an overview. J Interferon Cytokine Res. 2009;29: 313-326.

32. Grieb G, Merk M, Bernhagen J, et al. Macrophage migration inhibitory factor (MIF): a promising biomarker. Drug News Perspect. 2010;23:257-264
Clinical, Cosmetic and Investigational Dermatology

\section{Publish your work in this journal}

Clinical, Cosmetic and Investigational Dermatology is an international, peer-reviewed, open access, online journal that focuses on the latest clinical and experimental research in all aspects of skin disease and cosmetic interventions. All areas of dermatology will be covered; contributions will be welcomed from all clinicians and

\section{Dovepress}

basic science researchers globally. This journal is indexed on CAS. The manuscript management system is completely online and includes a very quick and fair peer-review system, which is all easy to use. Visit http://www.dovepress.com/testimonials.php to read real quotes from published authors. 